\title{
La auditoría en entorno COVID-19. Uso de tecnología y enfoque de riesgos
}

\section{Rotation of Public Managers. Problem of management or solution of political trust?}

INFORMACIÓN DEL

\section{ARTÍCULO}

Fecha de recepción: 6 de Septiembre de 2020.

Fecha de aceptación: 30 de Octubre de 2020 . $\overline{1 \text { Doctor en Gerencia, Universidad }}$ Yacambú. Docente-investigador, Universidad Politécnica Territorial Andrés Eloy Blanco-Venezuela.

E-mail: jesuscruz2008@gmail.com Código ORCID:

https://orcid.org/0000-0003-4916-2126
CITACIÓN: Cruz, J.E.(2020). La auditoría en entorno COVID-19. Uso de tecnología y enfoque de riesgos. Podium, 38, 67-86.

doi:10.31095/podium.2020.38.5

\section{ENLACE DOI:}

http://dx.doi.org/10.31095/podium.202 0.38 .5

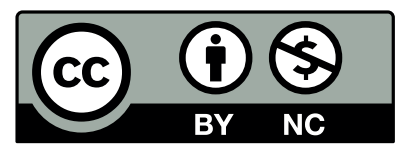

\section{Resumen}

El propósito de investigación es indagar las respuestas tecnológicas de la auditoría ante el impacto del Covid-19, en su enfoque de riesgos, pretendiendo explorar respuestas, comprender el uso tecnológico en auditoría y su utilidad en este entorno, y analizar el impacto del Covid-19 en el enfoque de auditoría. La metodología consiste en revisión bibliográfica de publicaciones científicas en Scopus y Google Scholar, análisis de contenido por categorización de páginas web de las consultoras KPMG, PWC, Deloitte, EY, y la construcción de macrocategorías. Los resultados evidencian respuestas tecnológicas, teletrabajo en auditoría remota, y auditoría continua, utilidad del uso tecnológico incorporando inteligencia artificial, automatización robótica, cloud, big data y analytics; el impacto del Covid-19 en los riesgos de auditoría se concentra en el error material en los estados financieros dada la valoración de la hipótesis de la continuidad del negocio en marcha, cambios en los métodos de trabajo del cliente e incorporación de tecnología.

\section{Palabras Clave:}

Covid-19, Auditoría, Auditoría Remota, Riesgos, Enfoque, Tecnología.

Clasificación JEL: G01; M42.

\begin{abstract}
The purpose of this research is to investigate the audit's technological responses to Covid-19's impact; it concerns its risk focus and attempts to explore responses and to understand its technological use in auditing as well as its usefulness in this environment; it also purports to analyze Covid-19's impact on auditing focus. The methodology used consisted of a bibliographical review of scientific publications in Scopus and in Google Scholar; it also consisted of web page content analysis by categorization of publication by consulting firms named KPMG, PWC, Deloitte, EY and the construction of macrocategories. Technological responses became evident from the research results, as did remote auditing tele-work as well as continuous auditing; usefulness of technological use of artificial intelligence, robotic automatization, cloud, big data and analytics also became evident from the results. Covid-19's impact on auditing risks concentrates on the material error in financial statements. These errors derive from the going concern hypothesis continuity valuation, as well as from the changes in the customer's working methods and technology incorporation.
\end{abstract}

\section{Keywords:}

Covid-19, Audit, Remote Audit, Risks, Focus, Technology.

JEL Classification: G01; M42.

$$
67
$$

\footnotetext{
PODIUM No. 38, Diciembre 2020, pp. 67-86

(C) Universidad Espíritu Santo - UEES

ISSN: 1390-5473 e-ISSN: 2588-0969
} 


\section{Introducción}

A modo de describir el entorno Covid-19 y su impacto en los riesgos económicos-financieros de las organizaciones, se centra la reflexión en la incertidumbre económica, considerando que se trata de los efectos de una crisis sanitaria producida por el coronavirus, extensa familia de virus que pueden causar enfermedades tanto en animales como en humanos (OMS, 2020). Covid 19, es la enfermedad infecciosa causada por el coronavirus descubierta a finales del año 2019.La incertidumbre impacta de diversas formas la actividad económica y toma de decisiones, por ejemplo, las organizaciones retrasan inversiones y la contratación incrementa sus costos de financiamiento al aumentar las primas de riesgo, otras empresas se paralizan o deciden cesar sus operaciones, algunas asumen el compromiso mediante la adaptación, y restructuración de

procesos y del modelo de negocio.

Entre los impactos de entorno Covid-19 vinculados a la incertidumbre económica está la necesidad de preservar la salud de su capital humano. La interrupción en la cadena de suministro por desvíos o retrasos, los cortes en las operaciones cuyas repercusiones van directas al cliente, la reducción de ventas con implicaciones en el flujo de caja y el cumplimiento de contratos, trabas en operaciones por la deslocalización e infraestructuras no disponibles, la postergación de viajes por restricciones a determinados destinos, y regulaciones, imponen nuevos retos en cuanto al cumplimiento de leyes y normas.
En entornos caracterizados por la incertidumbre económica y financiera, las organizaciones son afectadas en mayor o menor profundidad; las firmas de auditoría y los contadores públicos independientes, no escapan a esta realidad. La auditoría como profesión, ante esta pandemia entra en crisis, representando una oportunidad para evidenciar los avances al utilizar herramientas tecnológicas y de comunicación (TIC). Considerando este escenario incierto, cabe preguntar: ¿Qué respuestas tecnológicas tiene la auditoría ante el Covid-19?, ¿Qué utilidad ofrece el uso de tecnología en las auditorías realizadas en entorno Covid-19?, ¿Cuál es el impacto del Covid-19 en el enfoque de riesgos de auditoría? Despejar estas inquietudes es importante para comprender hacia donde se orienta la profesión.

Explorar las respuestas tecnológicas de la auditoría frente al Covid-19permite visualizar los efectos de la incorporación de los avances tecnológicos a los procedimientos del examen a los estados financieros, y al proceso de auditar en general. Su comprensión a través del análisis de los cambios informáticos en el tiempo, coadyuva a percibir la utilidad de la tecnología usada en auditoría para asumir el reto que impone esta pandemia.

Analizar el impacto en el enfoque de auditoría de riesgos nuevos o revisados surgidos a causa de Covid-19, es una contribución académica para futuros estudios, donde se evalúen escenarios de incertidumbre, valoren riesgos de negocios y planifiquen como auditar en estas circunstancias. 


\section{Revisión de literatura}

En el propósito de indagar las respuestas tecnológicas de la auditoría ante el impacto del Covid-19 en su enfoque de riesgos, se exponen estudios recientes vinculados al estudio, y un referencial teórico, sustentando este fenómeno de nueva data, en la teoría de la información en auditoría, propuesta por Mautz y Sharaf (1974), Boynton y Johnson (2005), y Konrath (2002).

La temática ha sido abordada desde la teoría de la información, que presupone que la procura de la auditoría radica en la fiabilidad y relevancia de la información financiera como input del proceso de toma de decisiones. Esta teoría incluye tres aspectos fundamentales: La producción de información financiera fiable, la producción de información financiera relevante para el proceso de decisión del inversor, y la credibilidad de la información financiera producida $\mathrm{y}$ divulgada (Machado y Marques, 2011).

Oneto (2020) refiere que la pandemia del Covid-19 no respeta fronteras, afecta tanto la salud de las personas como de las empresas y cuando una crisis de carácter global toca la puerta de estas, su estructura de gobierno corporativo se pone a prueba como herramienta para gestionar riesgos y salir adelante, es decir pone de manifiesto la gestión de riesgos para afrontar el impacto de la crisis en organizaciones. Estudios sobre incertidumbre realizados por Peng, Johnstone, y Christodoulou (2020) demuestran mediante modelo predictivo, el impacto asimétrico de divulgar noticias sobre la incertidumbre de los inversionistas. La percepción del riesgo aumenta en escenarios inciertos, pues las buenas noticias tienden a resolver más incertidumbre que las malas, y la incertidumbre puede revisarse al alza si la creencia previa de los inversores es lo suficientemente fuerte y la señal es lo suficientemente mala. En este entorno impactado por Covid-19, sin fecha de caducidad, la incertidumbre es una constante.

Desde una perspectiva constructivista, la pandemia ha desestructurado al mundo como se conocía hasta ahora, e invita a reflexionar nuevamente sobre la Contabilidad, su rol y sus posibles contribuciones para enfrentar algunos de los desafíos que trae la construcción de una infraestructura social y económica diferente (Rodríguez, 2020). Este planteamiento infiere la necesidad de avanzar, bien hacia una nueva normalidad o a la generación de realidades distintas a la conocida, mediante transición, adaptación o transformación.

Un esbozo generador de la cosmovisión contable sobre esta pandemia de PWC-España (2020), reseña que esta crisis es una ocasión única para auditar qué impacto ha tenido en la organización a corto plazo y cómo se ha gestionado, además de entender los potenciales riesgos de dicha gestión a mediano y largo plazo. Expresa que permite dar aseguramiento y obtener conclusiones sobre ámbitos de mejora a nivel de gestión de crisis y preparación ante estas situaciones desde distintos 
ángulos: personas y organización, procesos, sistemas, y reputación. Gestión y preparación ante la crisis, son la clave de las organizaciones para disminuir los efectos del coronavirus.

El impacto de esta crisis en la contaduría es de amplio espectro (Appelbaum, Budnik, y Vasarhelyi, 2020), el inicio de la pandemia ha resultado en una enorme presión en auditores y contadores para encontrar alternativas de formas de recopilar evidencia de auditoría y completar el compromiso. Se trata de los efectos causados en el cierre contable del ejercicio culminado al 31 de diciembre 2019 y la formulación de estados financieros para ese periodo, los cuales serán objeto de auditoría externa en pleno desarrollo de la crisis. Baskan (2020) concluye que dado que el tiempo transcurrido durante la pandemia de Covid-19 no es suficiente para realizar un análisis financiero, realizó su evaluación dentro del marco de la Norma Internacional de Auditoría 570 (IIASB, 2019), determinando de acuerdo con los resultados, que su efecto sobre el supuesto de negocio en marcha debería ser incluido en los informes y divulgaciones detalladas a fin de proporcionar una seguridad razonable a los usuarios de la información. Esta continuidad operativa debe ser evaluada con rigurosidad y revelada suficientemente, es lo recomendable para suministrar información financiera de calidad y generar confianza en los usuarios.

Lederman y Lederman (2015) mencionan que un marco teórico deficiente $o$ faltante, constituye una limitación crítica para los manuscritos construidos, por consiguiente, es de requerir la ampliación de los atributos y caracterizaciones teóricas, que, en este caso, están referidas a tres macrocategorias: (a) La Auditoría como respuesta tecnológica al entorno Covid-19, (b) Uso de la tecnología en la Auditoría. Su utilidad en el marco del Covid-19 y (c) Enfoque de riesgos de auditoría en un escenario Covid-19.

La Auditoría como respuesta tecnológica al entorno Covid-19

Desde una pregunta generadora cabe indagar ¿Qué aspectos del avance tecnológico ha asumido la auditoría? En primer escenario, se ubican los equipos o dispositivos de trabajo, los procedimientos para obtener las evidencias y la confiabilidad de ellas. No obstante, la práctica de la auditoría ha evolucionado a la par de las tecnologías de información TIC que usan las organizaciones para gestionar sus operaciones (Villamizar, 2016).

Una visión amplia apunta hacia la metodología de la auditoría, pues los avances tecnológicos y sus herramientas TIC, han logrado reducir tiempos de procesamiento de información y datos, mejoras en la interacción dialógica auditado-auditor y dedicación del tiempo ahorrado al análisis de información, son pilares de la construcción de un método de auditoría apropiado a los nuevos tiempos. Estos factores, en conjunto influyen en el proceso de auditoría, 
mediante la mejora de su eficiencia, a tal nivel que las auditorías pueden tener hoy en día un componente remoto y otra in situ, cuyas proporciones están relacionadas con los avances en tecnología de la información. Teeter, Alles, y Vasarhelyi (2010) han analizado la transformación de la auditoría tradicional en auditoría remota, centrándose en dos aristas: la comunicación interpersonal y el análisis de datos. Consideran que la auditoría remota es un proceso por el cual los auditores combinan tecnologías de la información y comunicación (TIC) con análisis de datos para evaluar e informar sobre la exactitud de los datos financieros y controles internos, recoger pruebas electrónicas e interactuar con la entidad auditada, independientemente de la ubicación física del auditor. Resalta en este concepto, la obtención de pruebas electrónicas y la interacción con el auditado desde donde se encuentre el auditor, es decir una auditoría a distancia.

La auditoría a distancia pretende facilitar el seguimiento de diversos riesgos sin necesidad de realizar una visita in situ a la ubicación física del cliente (Mendiola, 2008). ¿Existe alguna diferencia entre auditoría remota y auditoría continua? La diferencia puede estar en donde se encuentre el auditor. El proceso de auditoría continua surgió alrededor de 1989 mediante los esfuerzos de Vasarhelyi y Halper para medir y analizar el proceso de facturación en la empresa internacional AT\&T. De allí emergen diversas conceptualizaciones aportadas por Vasarhelyi y Halper (1991), Rezaee, Mcmickle, Sharbatoghlie, y
Elam (2004), Cruz (2013), entre otros.

Partiendo de estas conceptualizaciones, Cruz (2014a) extrae estas características: a) se trata de un proceso continuo; b) que requiere un soporte tecnológico; c) una estandarización de las operaciones para ser comparadas y d) una emisión de alertas sobre las desviaciones obtenidas de esa comparación. Para Álvarez (2017) la auditoría continua puede centrarse en cualquier tipo de información para la toma de decisiones, no sólo la presentación de informes de los estados financieros $\mathrm{o}$ temas comunes de auditoría.

\section{Las herramientas informáticas asumidas por la auditoría}

La auditoría ha incorporado los avances tecnológicos desde hace décadas, no en balde los aportes de Mattessich (2005) a la hoja electrónica de cálculo, combinando el sistema de partida doble y la matriz de contabilidad, siendo útiles en el establecimiento de las marcas de auditoría, apoyo en la supervisión de estas, y otorgar calidad en la documentación generada en los papeles de trabajo (Morales, 2019).

Al principio las organizaciones utilizaban los sistemas informáticos para el almacenamiento de datos y la capacidad de cálculo, incluyendo la información financiera, por ende, la función auditora se centró en procedimientos para evaluar el entorno de Procesamiento Electrónico de Datos (PED), haciendo hincapié en controles de acceso a los equipos de cómputo, clave 
de usuarios y resguardo físico de dispositivos. Luego el avance de la tecnología gira hacia la automatización de procesos o de las actividades del negocio, fortaleciendo el control interno organizacional, disminuyendo errores humanos. Este viraje lo aprovecha la auditoría para automatizar controles clave que permitan una revisión de mayor alcance y menor riesgo. A lo interno del proceso de auditoría se avanza en el uso de la tecnología para mejorar el muestreo mediante sistemas informáticos y los Procedimientos de Auditoría Asistidos por Computador (PAAC). Con el surgimiento de estos soportes informáticos, la auditoría se beneficia al incorporarlo a sus actividades de planificación, control de la ejecución y seguimiento, logrando optimizar los tiempos de auditoría, minimizando la utilización de recursos físicos, financieros y humanos, sin obviar, el impulso 1 usar el XBRL (extensible Business Reporting Language) como lenguaje universal en la realización del reporte financiero $y$ análisis de información a través de Internet. Ahora con el salto cuántico de la tecnología hacia el tratamiento masivo de datos Big Data, la auditoría con sus procesos ya automatizados obtiene la información de la organización auditada y procede al análisis de excepciones para recabar evidencias digitales que sustenten la opinión independiente. Algunos apuntan a la cadena de bloques, blockchain, como sustituto de la auditoría, no obstante, argumenta Cruz (2018), lo novedoso del disruptivo blockchain, es que se enfoca en la verificación de las transacciones, trae mejoras al proceso de datos $\mathrm{y}$ ficheros o archivos de información, agregando robustecimiento a la verificación de transacciones, pero no sustituye el juicio profesional, la valoración de riesgos y evidencias.

Uso de la tecnología en la Auditoría. Su utilidad en el marco del Covid-19

La auditoría ha tenido una transformación posibilitada por la tecnología, siendo ahora en tiempos de Covid-19, la respuesta de la auditoría externa e interna para asumir la profesión. La pandemia acelerará esta transformación dada la necesidad de brindar mejores prácticas profesionales (Deloitte, 2016). La auditoría remota como respuesta a la exigencia empresarial de un examen independiente de los estados financieros, no requiere la permanente presencia in situ del auditor, pues se efectúa a distancia, apoyándose en las herramientas tecnológicas de información y comunicación. Una actuación profesional de este estilo disminuye el riesgo de contagio por coronavirus de auditados y auditor. No obstante, incrementa el riesgo de auditoría, por el impacto económicofinanciero de la crisis en la organización. La auditoría continua a lo interno de las organizaciones es la implementación de herramientas tecnológicas al proceso de evaluación de los controles en tiempo real, a modo continuo, así como revisión de operaciones para obtener evidencias de posibles excepciones y emitir el respectivo informe de auditoría interna, específico a la actividad auditada, preciso, puntual y oportuno. Por lo general, el auditor debe estar en las 
instalaciones de la organización, sin embargo, puede aprovechar el bagaje profesional obtenido ejerciendo la auditoría interna continua y hacerlo de forma remota, para disminuir el riesgo sanitario de contraer el coronavirus. La auditoría remota o a distancia, asumiendo las capacidades tecnológicas de la auditoría interna continua, y viceversa, constituyen una herramienta efectiva para el ejercicio profesional en tiempos de pandemia. A tal efecto, los organismos reguladores de la profesión han publicado documentos técnicos referentes a la auditoría a distancia, entre ellos The Institute of Internal Auditors (IIA), quien publicó la investigación de los autores Litzenberg y Ramírez (2020). Esto implica una transformación de la profesión tal como lo manifiesta Pérez (2020), la auditoría con el salto tecnológico actual, tiene una oportunidad de mejora, en cuanto sea capaz de utilizar conjuntos y análisis de datos más grandes, posibilitará comprender mejor el negocio, identificar con más exactitud áreas de riesgo, ofrecer mayor cobertura y proporcionar más valor añadido.

Mirar hacia el futuro de la profesión implica que hay tecnologías florecientes prometedoras en el área de auditoría, incluyendo el uso de transmisión de video en vivo, realidad aumentada $\mathrm{y}$ virtual, vehículos aéreos no tripulados (drones) e inteligencia artificial, entre otros (Litzenberg y Ramírez, 2020).

\section{Enfoque de riesgos de auditoría en un escenario Covid-19}

El enfoque de riesgos en auditoría
(RA), se basa en un modelo donde existe un riesgo inherente a toda organización (RI) y un riesgo de control (RC) referido a la efectividad o no del control interno, aunado a un riesgo de detección (RD) correspondiente a la planificación del trabajo para obtener evidencias, por consiguiente, la responsabilidad de este último recae en los auditores. La expresión matemática del modelo es $\mathrm{RA}=$ $\mathrm{RI} \times \mathrm{RC} \times \mathrm{RD}$, de donde se deriva que $\mathrm{RD}=\mathrm{RA} /(\mathrm{RI} \times \mathrm{RC})$. El riesgo de detección consiste en dividir el riesgo de auditoría (RA) que significa el nivel aceptable de riesgo para esa auditoría, entre el riesgo inherente a la organización y sus ciclos transaccionales (RI), multiplicado por el riesgo de control (RC) que representa la efectividad del control interno en cuanto a confiabilidad de información, eficiencia de operaciones y cumplimiento de leyes y normativas. Estos riesgos, representan los componentes asociados a la auditoría; inherente -riesgos asociados con la naturaleza de la empresa, cuenta o transacción-; de control -riesgo de que los controles sobre la empresa, cuenta o transacción operen sin efectividad-; y de detección-riesgo de que los procedimientos del auditor no detecten los aspectos importantes que pueden afectar la empresa, cuenta o transacción (Cruz, 2014b). Para Mantilla (2002) este modelo matemático es producto de la excesiva subjetividad, el tratamiento aislado de riesgos en un mundo apostando a la integralidad, un enfoque extremadamente reducido, poca influencia para toma de decisiones de auditoría, y utilidad para sistemas no computarizados. Por ello, en la práctica 
las firmas auditoras incorporan el riesgo del negocio al modelo, dado que los objetivos de la auditoría varían con el cambio en entornos de negocios, y las técnicas de auditoría se modifican con los cambios en los objetivos de auditoría.

Respecto a las metodologías de trabajo, KPMG (2020) maneja un enfoque de auditoría proactiva, orientada a aportar soluciones, basada en un entendimiento del negocio, sistemas y riesgos. La auditoría centrada en riegos de negocio y en aquellos procesos críticos en las áreas que pudieran afectar la consecución de sus objetivos y la credibilidad de la información financiera, asegura consistencia, calidad, uso de tecnología y coordinación global a lo largo del proceso. En PWC (2020) su enfoque está basado en la sólida comprensión del negocio, sector en que opera y diferentes entornos regulatorios y geográficos donde realizan operaciones. Este conocimiento asegura que los diferentes riesgos que afectan al negocio constituyan la base de evaluación de riesgos de auditoría, y ayuda a definir el alcance y diseño del examen que dé respuestas a dichos riesgos. La evaluación y prueba de los controles en la empresa es un elemento esencial en este enfoque.

En Deloitte (2017, 2020), su enfoque es centrado en el riesgo con foco especial en la comprensión del negocio, la identificación de riesgos específicos y el desarrollo del plan de auditoría que responda a estos riesgos y a las circunstancias del cliente. La tecnología de Engagement Management System (EMS) permite a los profesionales enfocar sus esfuerzos en riesgos y cuentas materiales, mayor atención a la calidad, provee a los auditores la capacidad de crear, conducir y documentar el trabajo y ejercer mejor criterio profesional en determinar un enfoque específico para cada cliente. En EY (2020), la metodología de trabajo (Global Audit Methodology), a diferencia de otras metodologías usadas, está orientada al análisis y prueba de los controles que las compañías poseen, para reducir riesgos de errores en las representaciones y revelaciones hechas en los estados financieros, proporcionando un mayor valor a los clientes.

\section{Metodología}

Esta es una investigación cualitativa, con base documental e interpretativa de la realidad, partiendo de una revisión bibliográfica, de artículos científicos disponibles en la base de datos Scopus y Google Scholar, publicados entre las fechas comprendidas desde el 01 de enero y 20 de agosto de 2020. El criterio de selección es la literatura de reciente data, dado lo novedoso de la crisis sanitaria y sus efectos económicos-financieros, para ello, se realizaron búsquedas booleanas en los términos Auditoría + Covid-19. A efectos de sustentar la investigación en el marco de la teoría de la información en auditoría se construyeron las macrocategorías orientadoras como indican Lederman y Lederman (2015). Luego, se abordó el análisis de contenido inserto en páginas Web de cuatro firmas auditoras: KPMG, PWC, DELOITTE y EY. Con esta información se construyeron las matrices y se conformaron categorías de análisis y 
Tabla 1.

Matriz de Análisis de contenido

\begin{tabular}{|l|c|c|c|}
\hline Macro categoría & \multicolumn{2}{|c|}{} \\
\hline Unidad de Muestreo & Unidad de Análisis & Unidad de Contexto & $\begin{array}{c}\text { Categoría de } \\
\text { Análisis }\end{array}$ \\
\hline & & & \\
\hline
\end{tabular}

Fuente: Elaboración propia.

síntesis, relacionadas en el proceso deductivo-inductivo, para dar paso a la interpretación teórica global (Hoyos, 2000).

Se utilizó el análisis de contenido por categorización (Aigneren, 1999). Para ello, se identifican las unidades de análisis previstas: de muestreo, registro y contexto. La matriz diseñada (Tabla 1), ubica las macrocategorías y unidades de análisis,

\section{Tabla 2}

Matriz de Análisis de Contenido. Respuestas Tecnológicas

\begin{tabular}{|c|c|c|c|}
\hline \multirow{2}{*}{$\begin{array}{l}\text { Macro categoría } \\
\text { Unidad de Muestreo }\end{array}$} & \multicolumn{3}{|c|}{ Respuestas tecnológicas de la auditoría ante el Covid $\quad-19$} \\
\hline & Unidad de Análisis & Unidad de Contexto & $\begin{array}{c}\text { Categoría de } \\
\text { Análisis }\end{array}$ \\
\hline KPMG & $\begin{array}{l}\text { COVID -19: ¿Cuáles } \\
\text { son las } \\
\text { responsabilidades del } \\
\text { auditor? }\end{array}$ & $\begin{array}{l}\text { Trabajo en modalidad remota. } \\
\text { Nuestra cultura de trabajo } \\
\text { abarca la modalidad remota y } \\
\text { tenemos la capacidad para que } \\
\text { nuestros profesionales trabajen } \\
\text { desde sus hogares si es } \\
\text { necesario (Garcés, 2020). }\end{array}$ & $\begin{array}{l}\text { Auditoría } \\
\text { Interna } \\
\text { Auditoría } \\
\text { Remota }\end{array}$ \\
\hline \multirow{2}{*}{$P W C$} & PWC-Venezuela & Usar la tecnología $\frac{\text { de manera }}{\text { óptima. }}$ & $\begin{array}{c}\text { Auditoría a } \\
\text { Distancia }\end{array}$ \\
\hline & $\begin{array}{c}\text { ¿Cómo el COVID -19 } \\
\text { puede impactar tu } \\
\text { negocio? }\end{array}$ & $\begin{array}{l}\text { Teletrabajo -Home Office } \\
\text { Navegador PwC Covid -19 } \\
\text { (PWC, 2018) }\end{array}$ & $\begin{array}{l}\text { Auditoría } \\
\text { Interna }\end{array}$ \\
\hline Deloitte & \begin{tabular}{|l|} 
EL FUTURO DEL TRABAJO \\
Formas de trabajar para \\
mantenerse y triunfar en \\
tiempos inciertos
\end{tabular} & $\begin{array}{c}\text { Realizar todo el trabajo de } \\
\text { forma virtual }, \text { como una forma } \\
\text { de responder ante el brote del } \\
\text { virus. }\end{array}$ & $\begin{array}{l}\text { Auditoría } \\
\text { Virtual }\end{array}$ \\
\hline$E \boldsymbol{Y}$ & $\begin{array}{l}\text { COVID -19: cuatro } \\
\text { consejos de } \\
\text { planificación para } \\
\text { tener un trabajo } \\
\text { remoto exitoso }\end{array}$ & $\begin{array}{l}\text { Cada vez más, el teletrabajo y } \\
\text { el uso de la tecnología móvil } \\
\text { permiten que el personal elija } \\
\text { cuándo y cómo trabajar, } \\
\text { ampliando nuestra cultura de } \\
\text { colaboración, empoderamiento } \\
\text { y flexibilidad. }\end{array}$ & $\begin{array}{l}\text { Auditoría } \\
\text { Remota }\end{array}$ \\
\hline
\end{tabular}

Fuente: Elaboración propia. muestreo y de contexto en cada renglón, y la categoría de análisis, distinguiendo las respectivas unidades, para categorizar mediante reducción interpretativa, inferencial y conceptual de dichas unidades. La unidad de registro, se identifica mediante el subrayado en la unidad de contexto.

Posteriormente, se procede a la elucidación, interrelacionando expresiones 
de las unidades de muestreo, referentes teóricos e interpretación del autor, generando la triangulación de fuentes como aporte a la validación de la investigación.

\section{Resultados}

Del análisis de contenido de 15 documentos ubicables en páginas web de

Tabla 3.

Matriz de Análisis de Contenido. Utilidad en el USO de Tecnología Covid-19

\begin{tabular}{|c|c|c|c|}
\hline Macro categoría & \multicolumn{3}{|c|}{ Uso de la tecnología en la Auditoría. Su utilidad en el marco del Covid-19 } \\
\hline $\begin{array}{l}\text { Unidad de } \\
\text { Muestreo }\end{array}$ & Unidad de Análisis & Unidad de Contexto & $\begin{array}{c}\text { Categoría de } \\
\text { Análisis }\end{array}$ \\
\hline KPMG & $\begin{array}{l}\text { COVID -19: ¿Cuáles son } \\
\text { las responsabilidades del } \\
\text { auditor? } \\
\text { Auditoría Interna: dosis de } \\
\text { objetividad y perspectiva } \\
\text { ante el virus }\end{array}$ & $\begin{array}{c}\text { La tecnología se convierte en una } \\
\text { de las principales herramientas } \\
\text { del auditor para el desarrollo de sus } \\
\text { procedimientos en la búsqueda } \\
\text { de resultados más eficientes, } \\
\text { en cuanto al cubrimiento y la } \\
\text { oportunidad de los resultados. } \\
\text { Es de importancia que el } \\
\text { auditor cuente con ciertos } \\
\text { conocimientos relacionados } \\
\text { con el uso de procedimientos } \\
\text { automatizados como soporte a } \\
\text { la función de auditoría, encaminados } \\
\text { al manejo masivo de datos }\end{array}$ & Automatización \\
\hline$P W C$ & $\begin{array}{l}\text { Moviéndose a la velocidad } \\
\text { de la innovación. Las } \\
\text { herramientas y talentos } \\
\text { fundamentales de una } \\
\text { Auditoría Interna potenciada } \\
\text { por la tecnología. } \\
\text { Confianza en el futuro. Lo } \\
\text { humano y la M áquina } \\
\text { colaborando en la } \\
\text { Auditoría. }\end{array}$ & $\begin{array}{c}\text { Hoy en día, la Auditoría } \\
\text { Interna sólo está expuesta a un } \\
\text { número limitado de } \\
\text { tecnologías, como la } \\
\text { planificación de recursos } \\
\text { empresariales (ERP), cloud, } \\
\text { big data, analytics. Pero la } \\
\text { lista de tecnologías que la } \\
\text { Auditoría Interna debe } \\
\text { entender está creciendo } \\
\text { rápidamente. } \\
\text { Veinte años después, las } \\
\text { organizaciones están explorando el } \\
\text { el uso de la inteligencia artificial } \\
\text { (IA), por lo que la auditoría } \\
\text { enfrenta un cambio en su actuar. } \\
\text { La tecnología ha mejorado la } \\
\text { visión y la calidad de las } \\
\text { auditorías. Ahora, las } \\
\text { máquinas inteligentes nos dan } \\
\text { el potencial de redefinir } \\
\text { completamente la auditoría }\end{array}$ & $\begin{array}{c}\text { Cloud } \\
\text { Big Data, } \\
\text { Analytics }\end{array}$ \\
\hline
\end{tabular}

Fuente: Elaboración propia. 
Tabla 3.

Matriz de Análisis de Contenido. Utilidad en el USO de Tecnología Covid-19

\begin{tabular}{|c|c|c|c|}
\hline Macro categoría & \multicolumn{3}{|c|}{ Uso de la tecnología en la Auditoría. Su utilidad en el marco del Covid-19 } \\
\hline $\begin{array}{l}\text { Unidad de } \\
\text { Muestreo }\end{array}$ & Unidad de Análisis & Unidad de Contexto & $\begin{array}{c}\text { Categoría de } \\
\text { Análisis }\end{array}$ \\
\hline Deloitte & $\begin{array}{l}\text { La innovación en auditoría } \\
\text { asume las analíticas. } \\
\text { La ruta de la inteligencia } \\
\text { artificial (Davenport, 2020). } \\
\text { El inexplorado poder de la } \\
\text { "Auditoría Interna Digital" }\end{array}$ & \begin{tabular}{|c|} 
El auditor podría escoger \\
analizar todos los elementos \\
contenidos en ciertas poblaciones, \\
no solo una muestra de ellos. \\
Y los sistemas que automatizan \\
algunas tareas también pueden \\
facilitar esto. Deloitte, por \\
ejemplo, está usando una \\
herramienta para la extracción \\
automatizada de determinaciones \\
de los contratos para acelerar ese \\
proceso y revisar más documentos \\
con menos labor humana. \\
En los últimos años, las \\
capacidades de la auditoría interna \\
digital han aumentado \\
significativamente. Por ejemplo, \\
herramientas como la automatización \\
robótica de procesos (RPA, por sus \\
siglas en inglés) permiten que los \\
procesos que requieran mucho \\
trabajo y sean propensos a errores, \\
se terminen en cuestión de minutos.
\end{tabular} & $\begin{array}{c}\text { Automatización } \\
\text { Robótica }\end{array}$ \\
\hline$E \boldsymbol{Y}$ & $\begin{array}{l}\text { COVID -19: cuatro consejos } \\
\text { de planificación para tener } \\
\text { un trabajo remoto exitoso } \\
\text { Innovación en auditoría }\end{array}$ & $\begin{array}{l}\text { En medio del contexto del } \\
\text { Coronavirus (COVID-19) a nivel } \\
\text { mundial, en EY promovemos } \\
\text { hacer uso de la tecnología } \\
\text { para asegurar la salud de } \\
\text { nuestros colaboradores y, } \\
\text { a la vez, seguir comprometidos } \\
\text { con los clientes y sus desafíos } \\
\text { El aumento exponencial en la } \\
\text { cantidad y calidad de los datos } \\
\text { disponibles ha abierto nuevas } \\
\text { posibilidades de cómo los } \\
\text { equipos de auditoría pueden } \\
\text { utilizar los datos. } \\
\text { Las técnicas de inteligencia } \\
\text { artificial (IA), principalmente } \\
\text { en el aprendizaje automático, } \\
\text { permiten ver los datos mediante } \\
\text { el reconocimiento avanzado de } \\
\text { patrones y aprovechar esta } \\
\text { funcionalidad para ayudar } \\
\text { digitalmente a los auditores. }\end{array}$ & $\begin{array}{c}\text { Inteligencia } \\
\text { Artificial }\end{array}$ \\
\hline
\end{tabular}

Fuente: Elaboración propia. 
las firmas de auditoría, en la Tabla 2 se exploran las respuestas tecnológicas de la auditoría a la luz del escenario Covid-19.

Las respuestas tecnológicas de la auditoría ante el Covid-19 se centran en producir herramientas orientadas a fortalecer las unidades de auditoría interna de las organizaciones auditadas de manera independiente o externa. La auditoría interna da respuestas tecnológicas a un entorno de coronavirus mediante la auditoría continua, cuya característica esencial es el uso de la tecnología en las funciones auditoras a fin de mejorar su eficiencia, rapidez y confiabilidad.

En auditoría externa o independiente de estados financieros, las firmas de auditoría avanzan en la incorporación de tecnología de vanguardia, haciendo énfasis en aplicar lo recomendado a sus clientes, el teletrabajo, trabajo remoto, a distancia o virtual. La auditoría remota o a distancia requiere capacidades tecnológicas, las cuales han sido incorporadas, adaptadas y desarrolladas por estas firmas auditoras.

Para Litzenberg y Ramírez (2020), la auditoría remota no es una solución única. No es un reemplazo para una auditoría en persona. Sin embargo, como parte de un programa de garantía de varios años, la auditoría remota puede desempeñar un papel y proporcionar una garantía cuando circunstancias especiales impiden que las operaciones se realicen de forma habitual.

En la Tabla 3 se presentan los resultados asociados con la comprensión del uso de la tecnología en la auditoría y su utilidad en el marco del Covid-19.
El uso de la tecnología en auditoría en este tiempo, por parte de las firmas auditoras, se ha enfocado en la automatización robótica e inteligencia artificial, permitiendo examinar extensos volúmenes de transacciones, el análisis masivo de datos, y la ampliación del muestreo, minimizando riesgos de incorrecciones materiales en los estados financieros. También trabajan con Cloud, Big Data, Analytics, obteniendo provecho de estas innovaciones informáticas. Para García y Navallas (2020) utilizar Big Data y Analytics hace posible realizar pruebas de auditoría cubriendo un porcentaje casi total de la población, mitigando riesgos y generando mayor confianza en la prueba realizada, reducir costos y tiempo, incrementando la productividad y facilitando la identificación de asuntos claves.

Opina Pérez (2020) que la tecnología difícilmente podrá definir la estrategia de auditoría, cubrir los juicios de valor o tener la capacidad para tomar decisiones con base a los resultados obtenidos en los procedimientos. Recomienda aprovechar la evolución tecnológica y las tecnologías derivadas, para transformar la auditoría tradicional en auditoría avanzada; es decir, utilizar los avances tecnológicos como herramienta soporte de la función auditora. Desde luego, la innovación digital y mantener la relación con los clientes en las distintas fases del trabajo, asegura eficiencia, competitividad y control de riesgos. La tecnología permite el conocimiento y entendimiento del negocio y así puede valorar los riesgos adecuadamente y ser más eficientes en un entorno global. 
Como corolario se puede afirmar la reconversión de las firmas KPMG, PWC, Deloitte y EY, mediante la transformación digital de sus procesos, al aplicar dos estrategias: Adquirieron empresas especializadas en branding, marketing digital y tecnología e incorporaron profesionales 4.0 para desarrollar sus propios equipos $\mathrm{y}$ herramientas. La utilidad de la tecnología en la auditoría se plasma en el desarrollo de estas firmas, las cuales permearán aguas abajo hacia pequeñas y medianas auditoras y contadores públicos independientes es su saber-hacer.

Analizar el impacto en el enfoque de auditoría de riesgos nuevos o revisados que han surgido a causa de Covid-19

Los riesgos de auditoría de alto impacto, generados por el Covid-19, se clasifican en dos grupos (ver Tabla 4): (a) Error material en los estados financieros producto de la continuidad del negocio en marcha, hechos posteriores al cierre, limitaciones inherentes a la función auditora, modificaciones en la entidad auditada debido al Covid-19, estimaciones contables afectadas por la incertidumbre y (b) Riesgos tecnológicos.

El enfoque de auditoría es impactado por un riesgo de error material en los estados financieros, debido a la valoración de la continuidad del negocio en marcha, ya que la pandemia aumenta la incertidumbre en las empresas y sus proyecciones sobre la evaluación del supuesto de su continuidad operativa, dada la restricción en el flujo de caja, incumplimiento de contratos, y disminución de ingresos. La Norma Internacional de Auditoría-NIA 570 (IIASB, 2019) requiere que el auditor actué con escepticismo profesional

Tabla 4.

Matriz de Análisis de Contenido. Impacto del Covid-19 en el riesgo de auditoría

\begin{tabular}{|c|c|c|c|}
\hline Macro categoría & \multicolumn{3}{|c|}{ Impacto del Covid -19 en el enfoque de riesgos de auditoría } \\
\hline Unidad de Muestreo & Unidad de Análisis & Unidad de Contexto & $\begin{array}{c}\text { Categoría de } \\
\text { Análisis }\end{array}$ \\
\hline KPMG & $\begin{array}{l}\text { COVID -19: ¿Cuáles } \\
\text { son las } \\
\text { responsabilidades del } \\
\text { auditor? }\end{array}$ & $\begin{array}{l}\text { La efectividad de un enfoque de } \\
\text { auditoría basado en riesgos } \\
\text { depende, en gran medida, de la } \\
\text { capacidad del auditor para } \\
\text { identificar los riesgos de error } \\
\text { material en los estados } \\
\text { financieros debido a 'error o } \\
\text { fraude' y su adecuada } \\
\text { evaluación. }\end{array}$ & $\begin{array}{l}\text { Error Material } \\
\text { o Fraude }\end{array}$ \\
\hline$P W C$ & Auditoría & $\begin{array}{l}\text { Su enfoque de auditoría se basa } \\
\text { en la identificación oportuna de } \\
\text { riesgos, lo que permite } \\
\text { enfocarse en las áreas que } \\
\text { requieren un mayor desarrollo } \\
\text { y utilizar los recursos de la } \\
\text { manera más eficiente. }\end{array}$ & Error Material \\
\hline
\end{tabular}

Fuente: Elaboración propia. 
Tabla 4.

Matriz de Análisis de Contenido. Impacto del Covid-19 en el riesgo de auditoría

\begin{tabular}{|c|c|c|c|}
\hline Macro categoría & \multicolumn{3}{|c|}{ Impacto del Covid -19 en el enfoque de riesgos de auditoría } \\
\hline Unidad de Muestreo & Unidad de Análisis & Unidad de Contexto & $\begin{array}{c}\text { Categoría de } \\
\text { Análisis }\end{array}$ \\
\hline Deloitte & $\begin{array}{c}\text { Navegando los } \\
\text { impactos del COVID } \\
19\end{array}$ & $\begin{array}{l}\text { El análisis del libro mayor y la } \\
\text { revisión final, pueden } \\
\text { enfocarse en áreas claves de } \\
\text { riesgo en finanzas, debido al } \\
\text { tiempo y la disponibilidad de } \\
\text { recursos. } \\
\text { Las limitaciones de capacidad y } \\
\text { la disponibilidad de los } \\
\text { archivos de trabajo plantearán } \\
\text { problemas para los auditores } \\
\text { internos y externos. } \\
\text { Conectar con los equipos de } \\
\text { auditoría interna y externa, para } \\
\text { garantizar que exista un plan } \\
\text { para probar los procedimientos } \\
\text { en un ambiente remoto. } \\
\text { Comprender el impacto que el } \\
\text { COVID-19 tendrá en el } \\
\text { enfoque de prueb a de su auditor } \\
\text { (naturaleza, tiempo, extensión } \\
\text { de pruebas). } \\
\text { Discutir con los auditores } \\
\text { externos desde un inicio el } \\
\text { tratamiento contable para las } \\
\text { áreas claves de los estados } \\
\text { financieros que tendrán } \\
\text { impactos por el COVID -19. } \\
\text { Si el tiempo lo permite, } \\
\text { adelantar los controles y } \\
\text { requisitos de auditoría }\end{array}$ & Error Material \\
\hline$E Y$ & $\begin{array}{l}\text { COVID -19: } r \text { Cinco } \\
\text { pasos para defenderse } \\
\text { de los atacantes } \\
\text { cibernéticos } \\
\text { oportunistas }\end{array}$ & $\begin{array}{l}\text { El auge del teletrabajo aumenta } \\
\text { los riesgos. } \\
\text { El aumento del trabajo a } \\
\text { distancia incrementa la carga } \\
\text { de los equipos de apoyo de IT. }\end{array}$ & $\begin{array}{c}\text { Riesgos } \\
\text { Tecnológicos }\end{array}$ \\
\hline
\end{tabular}

Fuente: Elaboración propia.

cuando de revisar las hipótesis y estimaciones efectuadas por el cliente. fundamentos de la gerencia sobre el negocio en marcha se trata, así como obtención de evidencias que soportan las

Por ello, sugiere a los auditores, la obtención de evidencia suficiente $\mathrm{y}$ 
adecuada a fin de llegar a la conclusión de que los potenciales efectos del coronavirus están acertadamente incorporados en estas proyecciones. Para Eyzaguirre (2020) debe tenerse en cuenta que algunas circunstancias podrían orientar al auditor a dar especial énfasis a un determinado enfoque, como el de procedimientos sustantivos, o combinando la aplicación de pruebas de controles y procedimientos sustantivos, destacando la Norma Internacional de Auditoría 240 (IIASB, 2019), correspondiente al riesgo inevitable de que no puedan detectarse algunas incorrecciones materiales en los estados financieros, incluso aunque la auditoría se haya planificado y ejecutado adecuadamente de conformidad con las NIA, debido a las limitaciones inherentes a una auditoría.

La Norma Internacional de Auditoría 315 (IIASB, 2019), riesgos de incorrección material, debido a fraude o error, que afecten los estados financieros, incluidas afirmaciones intrínsecas en los mismos; modificaciones sustanciales en la entidad cuyos estados financieros son materia de auditoría, que afecten el conocimiento y entorno de la entidad por el auditor, incluido su control interno. La Norma Internacional de Auditoría 540 (IIASB, 2019) de la naturaleza y fiabilidad de la información base para la realización de una estimación contable es variada, lo cual afecta el grado de incertidumbre, $\mathrm{y}$, a su vez, al riesgo de incorrección material en dicha estimación, inclusive la susceptibilidad a un sesgo de la dirección, intencionado o no, y la Norma Internacional de Auditoría 560 (IIASB, 2019) sobre los determinados hechos que ocurran con posterioridad a la fecha de los estados financieros, pueden afectarlos significativamente.

La migración de las entidades auditadas hacia el teletrabajo, la virtualización de sus procesos, en ocasiones obviando medidas de seguridad y protección adecuada de la información, aumentan los riesgos de ataques cibernéticos, pérdida de información, entre otros. Consecuentemente los auditores deben revisar las conclusiones en pruebas de controles de entornos informáticos, sistemas y redes, a fin de valorar potenciales errores materiales o de fraude en la información computarizada, automatizada y dispuesta en cloud data, y dropbox.

Existen otros riesgos asociados a la tecnología, aclara la Federación Argentina de Consejos Profesionales de Ciencias Económicas (2020):

Riesgo de que la infraestructura tecnológica no satisfaga la mayor demanda, debido al régimen de trabajo

Planificar y organizar con miembros del equipo de trabajo determinados horarios para acceder a plataformas o servidores remotos, en casos donde la infraestructura tecnológica no pueda suplir la alta demanda de trabajo remoto, ocasionando problemas de conexión, acceso a servidores, excesiva lentitud en el uso de aplicaciones, entre otros. De esa forma, no se estaría estresando la infraestructura de tecnología y cada colaborador podría acceder de mejor manera. 
Limitación en el uso de recursos tecnológicos, especialmente para pequeñas y medianas empresas

Darle a conocer al cliente la existencia de diferentes recursos tecnológicos desde software de nube (Dropbox, OneDrive, Google Drive, SharePoint), que pueden utilizarse como paliativos en caso de no contar con tecnología para trabajar en forma remota o no pueda utilizar la información disponible en los servidores. $\mathrm{El}$ auditor debe fomentar su uso por parte del cliente, utilizar procedimientos adicionales y complementarios para restringir datos e información, recomendar la asignación de claves de acceso a los archivos con destino a ser enviados fuera de la entidad, aupar el encriptado de estos archivos, inducir al cliente a tomar como precaución que se envíen en e-mails separados los archivos base con clave y las respectivas contraseñas para acceder a ellos, y usar doble medio de comunicación para aumentar la seguridad de los archivos enviados.

\section{Conclusiones}

Con base a los documentos analizados se concluye que la auditoría avanza en consonancia con el cambio tecnológico, el cual supone modificaciones en el enfoque de riesgos, dada la transformación en el negocio del auditado $\mathrm{y}$ en los procedimientos del auditor, atendiendo al postulado de la teoría de la información en auditoría. En el entorno Covid-19, la auditoría ha dado respuestas tecnológicas al fortalecer las unidades de auditoría interna en las organizaciones en su adaptación al proceso de auditoría continua y en la utilización del trabajo remoto en la auditoría externa.

La tecnología usada en auditoría ante el entorno Covid-19, por las firmas auditoras son inteligencia artificial (IA), automatización robótica, cloud, big data, analytics, entre otras, que permiten el análisis masivo de datos, e incrementa el muestreo y una mayor comprensión del negocio. KPMG incorporó nuevas capacidades tecnológicas para profundizar en el análisis de grandes volúmenes de datos con sistemas cognitivos aplicados a la auditoría. PWC incrementó la inversión en tecnología y transformó la firma, implantando nuevas herramientas y mejorando procesos para adaptar de forma eficiente su modo de trabajo y responder ante esta nueva realidad.

Deloitte se encuentra en transformación y adecuación a los cambios tecnológicos, indicando que la innovación puesta al servicio del auditor no es algo del futuro, sino del presente. Posee herramientas para extraer, ordenar y analizar la totalidad de las transacciones, convirtiendo a la auditoría en una revisión milimétrica a través de un análisis granular. En EY, la tecnología posibilita aplicar herramientas Big Data a la auditoría externa, ampliando notablemente el muestreo de auditoría, haciendo más precisa aún y fiable la información financiera.

La transformación digital de la auditoría en estas firmas es producto de la adquisición de empresas dedicadas al 
marketing online, branding y desarrollo de herramientas propias y sistemas tecnológicos. Este tiempo de Covid-19 es testigo presencial e impulsor de la reconversión de las auditoras.

El enfoque de riesgos en auditoría es impactado por posibles errores materiales en los estados financieros, dada la valoración de la continuidad del negocio en marcha, ante la incertidumbre en las empresas y sus proyecciones sobre este supuesto contable. La NIA 570 (IIASB, 2019) requiere que el auditor actué con escepticismo profesional al momento de revisar hipótesis y fundamentos de la gerencia sobre el negocio en marcha, así como obtención de evidencias que soportan las estimaciones efectuadas por el cliente. Tiene impacto el riesgo inevitable de no detección de algunas incorrecciones materiales en los estados financieros, producto de limitaciones inherentes a una auditoría (NIA 240), el riesgo de fraude y error por modificaciones en el entorno de la entidad auditada (NIA $315)$, el riesgo de incorrección material en estimaciones contables, incluyendo la susceptibilidad a un sesgo de la dirección, intencionado o no (NIA 540) y por hechos posteriores a la fecha de los estados financieros (NIA 560).

El trabajo remoto en clientes como en las firmas auditoras, conlleva un riesgo en el enfoque de auditoría debido a la incertidumbre, pérdida de información, ciberataques (Lovejoy, 2020), o que la infraestructura tecnológica no satisfaga la mayor demanda, ante esta modalidad de trabajo y limitaciones tecnológicas en pequeñas y medianas empresas.
El Covid-19 acelera la transformación digital de firmas auditoras y clientes, por la necesidad de darle continuidad al negocio, enfrentar la interrupción de cadena de suministros, restricción en los flujos de caja, e incumplimiento de contratos. Estos cambios permearán aguas abajo en pequeñas y medianas firmas, contadores públicos independientes, beneficiando la práctica profesional y regulaciones. Los auditores deben diseñar una adecuada respuesta en el tratamiento de los riesgos, utilizando su capacidad innovadora para definir procedimientos de auditoría que superen posibles limitaciones en el alcance de la auditoría, considerando esquemas de trabajo adoptados por clientes para mitigar riesgos de contagio del Covid-19.

\section{Referencias}

Aigneren, M. (1999). Análisis De Contenido. Una Introducción. Revista La Sociología en sus Escenarios, (3), 1-52.

Álvarez, V. (2017). Aplicación de un Modelo de Auditoría Continua Utilizando JD Edwards Enterprise One. INVESTIGATIO, (8), 31 - 49.

Appelbaum, D., Budnik, S. y Vasarhelyi, M. (2020) Auditing and Accounting During and After the COVID-19 Crisis. The CPA Journal. Recuperado de: https://www. cpajournal.com/2020/07/08/auditingand-accounting-during-and-after-the-covi d-19-crisis/

Baskan, T. (2020). Analyzing the going concern uncertainty during the period of Covid-19 pandemic in terms of independent auditor's reports. ISPEC International Journal of Social Sciences \& Humanities, 4(2), 28-42. https://doi.org/10.46291/ ISPECIJSSHvol4iss2pp28-42 
Boynton, W., y Johnson, R. (2005). Modern Auditing: Assurance services and the integrity of financial reporting, octava edición. New York: Wiley.

Cruz J. (2013). Auditoría Continua. Recuperado de: es.scribd.com/doc/139951538/Audito ria-Con tinua-05-2013?

Cruz, J. (2014a). Auditoría Continua como herramienta de control fiscal: Bases conceptuales para su aplicación en Fundacite-Lara. TEACS, (15), 67-80.

Cruz, J. (2014b). Consideraciones sobre el muestreo en auditoría: selección de evidencias e impacto en el riesgo de detección. Revista CICAG, 11(2), 119-135.

Cruz, J. (2018). La Criptomoneda y el subyacente registro contable distribuido (LDT). Una aproximación a la contabilidad triangular. Holopraxis, 2(2), 15-34.

Davenport, T. (2020). La innovación en auditoría asume las analíticas. La ruta de la inteligencia artificial. Deloitte. Recuperado de: https:// www2.deloitte.com/co/es/pa ges/audit/articles/innovacio_auditoria_an aliticas.html

Deloitte. (2016). Reglamento Interno Prácticas Profesionales. Recuperado de: https: //www2.deloitte.com/content/dam/Deloit te/cl/Documents/audit/cl-Reglamento $\% 2$ 0Interno\%20Profesional_12.01.17.pdf

Deloitte. (2017). El inexplorado poder de la "Auditoría Interna Digital". Recuperado de: https://www2.deloitte.com/content/ dam/ Deloitte/pa/Documents/audit/2018 _PA_AA_Explotar_AA_Interna.pdf.

Deloitte. (2020). EL FUTURO DEL TRABAJO. Formas de trabajar para mantenerse y triunfar en tiempos inciertos. Recuperado de: https:// www2.deloitte.com/cl/es/ pages/about-deloitte/articles-covid19/fut uro-del-trabajo.html.
Deloitte. (2020). Navegando los impactos del

COVID 19. Recuperado de: https://www2. deloitte.com/content/dam/ Deloitte/cl/Documents/finance/cl-navega ndo-impactos-covid-19.pdf.

EY. (2020). COVID-19: cuatro consejos de planificación para tener un trabajo remoto exitoso. Recuperado de: https://www. ey.com/es_py/covid-19/covid-19-consejo s-trabajo-remoto

EY. (2020). Innovación en auditoría. Recuperado de: https://www.ey.com/es_ec/audit/innova tion

Eyzaguirre, D. (2020). Impacto del Covid-19 en el enfoque de la Auditoría de Estados Financieros. https://www.bakertilly.pe/ wp-content/uploads/Impacto-del-Covid-1 9-en-el-enfoque-de-la-Auditor $\% \mathrm{C3} \% \mathrm{AD}$ a-de-Estados-Financieros-DE-Mayo-202 $0 . p d f$

Federación Argentina de Consejos Profesionales de Ciencias Económicas. (2020) Guía sobre la Aplicación de las Normas de Contabilidad y Auditoría frente a los efectos del Covid-19. https://www.fac pce.org.ar/pdf/GuiaCovip-19-2.pdf

Garcés, M. (2020). COVID-19: ¿Cuáles son las responsabilidades del auditor? KPMG. Recuperado de: https:/home.kpmg/co/ es/home/insights/2020/05/covid-19-cuale s-son-las-responsabilidades-del-auditor.h tml

García, N., y Navallas, B. (2020). Auditoría y Data Analytics: valoración de los profesionales en España. AECA: Revista de la Asociación Española de Contabilidad y Administración de Empresas, (130), 15-18.

Hoyos, C. (2000). Un modelo para una investigación documental. Guía teórico- práctica sobre construcción de estados del arte con importantes reflexiones sobre la investigación. Medellín: Señal Editora. 
IIASB - International Auditing and Assurance Standards Board. (2019). Normas Internacionales de Auditoría. Federación Internacional de Contadores-IFAC. New York.

Konrath, L. (2002). Auditing: A risk analysis approach, 5ta edición. Cincinnati: South-Western.

KPMG. (2020). Auditoría Interna: dosis de objetividad y perspectiva ante el virus. Recuperado de:https:/home.kpmg/ve/es/ home/insights/2020/03/auditoria-internacovid-190.html

KPMG. (2020). KPMG ante la Covid 19. Nuestra respuesta. Recuperado de: https://home. kpmg/es/es/home/about/kpmg-ante-covid $-19 . \mathrm{html}$

Lederman, N., y Lederman, J. (2015). What is a theoretical framework? A practical answer. Journal of Science Teacher Education, 26, 593-597. https://doi.org/ 10.1007/s10972-015-944 3-2.

Litzenberg, R., y Ramírez, C. (2020). Auditoría remota para Covid-19 y demás. Implicaciones a corto y largo plazo. The Institute of Internal Auditors. Recuperado de: https://na.theiia.org/translations/Publi cDocuments/EHSKB-Remote-Auditing-f or-COVID-19-and-Beyond-Spanish.pdf

Lovejoy, K. (2020). COVID-19: Cinco pasos para defenderse de los atacantes cibernéticos oportunistas. EY. Recuperado de: https:// www.ey.com/es cl/consulting/covid-19-s teps-to-defend-against-opportunistic-cyb er-attackers

Machado, B., y Marques, J. (2011). En torno a las teorías justificativas de la auditoría. Partida Doble, (228). Recuperado de: http ://pdfs.wke.es/2/1/5/9/pd0000062159.pdf

Mantilla, S. (2002). De los riesgos de auditoría a los riesgos de negocio. El cambio de modelo. Revista Internacional Legis de Contabilidad y Auditoría, (12), 11-115.
Mattessich, R. (2005). A Concise History of Analytical Accounting: Examining the use of mathematical notions in our discipline. De Computis-Revista Española de Historia de la Contabilidad, 2(2), 123-153.

Mautz, R., y Sharaf, H. (1974). The philosophy of auditing, 7th edition. Sarasota: American Accounting Association.

Mendiola, M. (2008). Auditoría tecnológicamente avanzada: Auditoría a distancia y auditoría continua. Partida Doble, (204), 42-47.

Morales, F. (2019). Tecnología de la Información como herramienta de la auditoría. Revista Contaduría Pública. Disponible en:http:// contaduriapublica.org. $\mathrm{mx} / 2019 / 10 / 01 /$ tec nologia-de-la-informacion-como-herrami enta-de-la-auditoría/

Oneto, A. (2020). COVID-19: Continuidad del negocio, gestión de crisis y gobierno corporativo. Caracas: CAF.

Organización Mundial de la Salud-OMS. (2020). Preguntas y respuestas sobre la enfermedad por coronavirus (COVID-19). Recuperado de: https:// www.who.int/es/ emergencies/diseases/novel-coronavirus2019/advice-for-public/q-a-coronaviruses

Peng, Z., Johnstone, D., y Christodoulou, D. (2020). Asymmetric impact of earnings news on investor uncertainty. Journal of Business Finance \& Accounting, 47(1-2) 3-26, https://doi.org/10. 1111/jbfa.12428.

Pérez, I. (2020). Tecnología y auditoría: principales retos. Portal del Auditor. Instituto de Censores Jurados de Cuentas de España. Recuperado de: https://www.icjce.es/ tecnologia -auditoria-principales-retos.

PWC. (2018). Estudio del estado de la profesión de auditoría interna 2018. Moviéndose a la velocidad de la innovación. Las herramientas y talentos fundamentales de 
una Auditoría Interna potenciada por la tecnología. Recuperado de: https:// www.pwc.com/cl/es/publicaciones/assets /2018/Moviendose-a-la-velocidad-de-la-i nnovacion-web.pdf

PWC-España. (2020). COVID-19 Impacto y rol de las funciones de aseguramiento. Recuperado de: https://www.pwc.es/es/ covid/assets/impacto-y-rol-de-las-funcio nes-de-aseguramiento.pdf

PWC. (2020). Confianza en el futuro: Humano y la Máquina colaborando en la Auditoría. Recuperado de: https://www.pwc.com/ia/ es/publicaciones/human-and-machine-co llaboration-in-the-audit.html

PWC. (2020). ¿Cómo el COVID-19 puede impactar tu negocio? Recuperado de: https://www. pwc.ec/es/como-el-covid-19-puede-impa ctar-tu-negocio.html

PWC. (2020). Gestión de riesgos en tiempos de COVID-19. Recuperado de: https://www. pwc.com.ar/es/servicios/auditoria/risk-as surance-services/gestion-riesgo-tiemposcovid-19.html

Rezaee, Z., Mcmickle, P., Sharbatoghlie, A., y Elam, R. (2004). Auditoría continua: Construyendo capacidades para una auditoría automatizada. Revista Internacional Legis de Contabilidad y Auditoría, (18), 9-40.

Rodríguez, M. (2020). La Contabilidad en tiempos del COVID-19. Contabilidad y Auditoría, (51), 109-154.

Teeter, R., Alles, M., y Vasarhelyi, M. (2010). The Remote Audit. Journal of Emerging Technologies in Accounting, 7(1),73-88. https://doi.org/10.2308 /jeta.2010.7.1.73

Vasarhelyi, M., y Halper, F. (1991). The Continuous Audit of Online Systems. Auditing: A Journal of Practice and Theory, 10(1), 110-125
Villamizar, L. (2016). Propuesta de teletrabajo para la función de auditoría en una entidad de control fiscal colombiana. Tesis de Maestría en Administración. Facultad de Ciencias Económicas, Universidad Nacional de Colombia. 